\title{
The cube polynomial and its derivatives: the case of median graphs
}

\author{
Boštjan Brešar* \\ FEECS, University of Maribor \\ Smetanova 17, 2000 Maribor, Slovenia \\ bostjan.bresar@uni-mb.si \\ Sandi Klavžar ${ }^{\dagger}$ \\ Department of Mathematics, PeF, University of Maribor \\ Koroška cesta 160, 2000 Maribor, Slovenia \\ sandi.klavzar@uni-mb.si \\ Riste Škrekovski ${ }^{\ddagger}$ \\ Department of Mathematics, University of Ljubljana \\ Jadranska 19, 1111 Ljubljana, Slovenia \\ skreko@fmf.uni-lj.si
}

Submitted: Jun 21, 2001; Accepted: Dec 27, 2002; Published: Jan 10, 2003

MR Subject Classifications: 05C30,05A15,05C12

\begin{abstract}
For $i \geq 0$, the $i$-cube $Q_{i}$ is the graph on $2^{i}$ vertices representing $0 / 1$ tuples of length $i$, where two vertices are adjacent whenever the tuples differ in exactly one position. (In particular, $Q_{0}=K_{1}$.) Let $\alpha_{i}(G)$ be the number of induced $i$-cubes of a graph $G$. Then the cube polynomial $c(G, x)$ of $G$ is introduced as $\sum_{i \geq 0} \alpha_{i}(G) x^{i}$. It is shown that any function $f$ with two related, natural properties, is up to the factor $f\left(Q_{0}, x\right)$ the cube polynomial. The derivation $\partial G$ of a median graph $G$ is introduced and it is proved that the cube polynomial is the only function $f$ with the property $f^{\prime}(G, x)=f(\partial G, x)$ provided that $f(G, 0)=|V(G)|$. As the main application of the new concept, several relations that widely generalize previous such results for median graphs are proved. For instance, it is shown that for any $s \geq 0$ we have $c^{(s)}(G, x+1)=\sum_{i \geq s} \frac{c^{(i)}(G, x)}{(i-s) !}$, where certain derivatives of the cube polynomial coincide with well-known invariants of median graphs.
\end{abstract}

* Supported by the Ministry of Education, Science and Sport of Slovenia under the grant Z1-30730101-01.

${ }^{\dagger}$ Supported by the same Ministry under the grant 101-504.

${ }^{\ddagger}$ Supported by the same Ministry under the grant Z1-3219. 


\section{Introduction}

Median graphs present a natural generalization of trees and hypercubes. It is therefore not surprising that they have been independently introduced several times $[1,12,15]$. These graphs are closely related to distributive lattices and graph retracts; see Avann [1], Bandelt [2], and Duffus and Rival [5]. Mulder [12, 13] proved that a graph $G$ is a median graph if and only if $G$ can be obtained from $K_{1}$ by the so-called convex expansion procedure. A step in such procedure can, intuitively, be described as follows. Cover a graph $H$ with two subgraphs $H_{1}, H_{2}$ such that the intersection $X$ of the cover is convex, take the disjoint union of $H_{1}$ and $H_{2}$ and finally insert a natural matching between the two copies of $X$. A similar characterization of median graphs is due to Bandelt and van de Vel [3]. Namely, a connected graph is a median graph if and only if it can be obtained from hypercubes by a sequence of convex amalgamations, where a convex amalgamation consists of gluing together two graphs along isomorphic convex subgraphs. For a survey of known characterizations of median graph see [11] and for infinite median graphs consult [18]. Recently a surprising connection between median graphs and (bipartite or nonbipartite) triangle-free graphs was discovered that asserts, intuitively speaking, that there are as many median graphs as there are triangle-free graphs [8].

Let $G$ be a median graph with $n$ vertices, $m$ edges, and $k$ equivalence classes of $G$ with respect to the Djoković-Winkler $[4,20]$ relation $\Theta$. In [10] it was proved that $2 n-m-k \leq 2$, and that equality holds if and only if $G$ is a cube-free median graph. Earlier, Soltan and Chepoi [17, Theorem 4.2.(6)] proved that

$$
\sum_{i \geq 0}(-1)^{i} \alpha_{i}(G)=1
$$

where $\alpha_{i}(G)$ denotes the number of induced $i$-cubes of $G$. This result was later independently obtained in [16], where it was also proved that

$$
k=-\sum_{i \geq 0}(-1)^{i} i \alpha_{i}(G)
$$

Equality (1) presents a generalization of the well-known equality " $n-m=1$ " for trees, while (2) applied to trees says that " $k=m$ ", which is another characterizing property of trees. These two relations in turn imply the Euler-type formulas from $[9,10]$.

The main purpose of this paper is to present a general approach for obtaining such equalities. The corresponding results that generalize all the above equalities are given in the second part of the paper. To obtain these results, we introduce the cube polynomial of

a graph $G$ as $c(G, x)=\sum_{i>0} \alpha_{i}(G) x^{i}$. (For other graph polynomials see [6].) We first show some basic properties of the cube polynomial: the amalgamation, product, and expansion properties. In fact, any function $f$ with the amalgamation, and expansion properties is up to the factor $f\left(K_{1}, x\right)$ the cube polynomial. Weaker conditions suffice to reach similar conclusions if we restrict to the class of median graphs. Then we introduce the derivation graph $\partial G$ of a median graph $G$ and prove that the cube polynomial is the only function $f$ with the property $f^{\prime}(G, x)=f(\partial G, x)$ provided that its value at $x=0$ equals the number 
of vertices. In the last section we prove several relations for median graphs involving the cube polynomial. Among others we prove that for any $s \geq 0$ we have

$$
c^{(s)}(G, x)=\sum_{i \geq s} \frac{(-1)^{i-s}}{(i-s) !} c^{(i)}(G, x+1) .
$$

This equality in turn implies

$$
\theta_{s}(G)=s ! \sum_{i \geq 0}(-1)^{i-s}\left(\begin{array}{l}
i \\
s
\end{array}\right) \alpha_{i}(G),
$$

where $\theta_{s}(G)$ is the number of components of the $s$-derivation of the graph $G$ which by Proposition 8 equals the $s$-th derivative of $c(G, x)$ evaluated at $x=-1$. It is always satisfied that $\theta_{0}(G)=1$ and $\theta_{1}(G)=k$. Thus plugging $s=0$ and $s=1$ into (3) we obtain (1) and (2), respectively.

\section{Preliminaries}

Let $\mathcal{G}$ be the set of all finite graphs. Denote by $P_{n}$ the path on $n$ vertices, and by $K_{n}$ the complete graph on $n$ vertices. For $i \geq 0$ let $Q_{i}$ be the hypercube of dimension $i$, or shortly the $i$-cube. Its vertex set consists of all $0 / 1$ tuples of length $i$, two vertices being adjacent whenever the tuples differ in exactly one position. Note that $Q_{0}=K_{1}$ and $Q_{1}=K_{2}$.

The Cartesian product $G \square H$ of graphs $G$ and $H$ is the graph with vertex set $V(G) \times$ $V(H)$ in which the vertex $(a, x)$ is adjacent to the vertex $(b, y)$ whenever $a b \in E(G)$ and $x=y$, or $a=b$ and $x y \in E(H)$. It is easy to see that the Cartesian product of $i \geq 1$ copies of $K_{2}$ is the $i$-cube $Q_{i}$.

A subgraph $H$ of a graph $G$ is an isometric subgraph if $d_{H}(u, v)=d_{G}(u, v)$ for all $u, v \in V(H)$, where $d_{G}(u, v)$ denotes the length of a shortest path in $G$ from $u$ to $v$. A subgraph $H$ of a graph $G$ is convex if for any two vertices $u, v$ of $H$ all shortest paths between $u$ and $v$ in $G$ are already in $H$. Isometric subgraphs of hypercubes are called partial cubes. A graph $G$ is a median graph if there exists a unique vertex $x$ to every triple of vertices $u, v$, and $w$ such that $x$ lies simultaneously on a shortest $u, v$-path, a shortest $u, w$-path, and a shortest $w, v$-path. Median graphs are partial cubes, cf. [7, 13]. Let $\mathcal{M}$ denote the class of all median graphs.

Two edges $e=x y$ and $f=u v$ of $G$ are in the Djoković-Winkler [4, 20] relation $\Theta$ if $d(x, u)+d(y, v) \neq d(x, v)+d(y, u)$. Winkler [20] proved that a bipartite graph is a partial cube if and only if $\Theta$ is transitive. Consider for instance the $i$-cube $Q_{i}$. Let $e \in E\left(Q_{i}\right)$ and suppose that the ends of $e$ differ in position $r$, where $r \in\{1, \ldots, i\}$. Then $e$ is in relation $\Theta$ with precisely those edges of $Q_{i}$ whose ends differ in position $r$. Consequently, $\Theta$ is transitive on $E\left(Q_{i}\right)$ and consists of $i$ equivalence classes.

Let $\mathcal{I}_{n}=\{1, \ldots, n\}$. In the paper the inclusion-exclusion principle will be used, which for given sets $A_{1}, \ldots, A_{n}$ says that

$$
\left|A_{1} \cup A_{2} \cup \cdots \cup A_{n}\right|=\sum_{\mathcal{J} \subseteq \mathcal{I}_{n}}(-1)^{|\mathcal{J}|-1}\left|\bigcap_{i \in \mathcal{J}} A_{i}\right| .
$$

THE ELECTRONIC JOURNAL OF COMBINATORICS 10 (2003), \#R3 


\section{The cube polynomial}

For a graph $G$, let $\alpha_{i}(G)$ denote the number of induced $i$-cubes of $G$. Note that $\alpha_{0}(G)=$ $|V(G)|$ and $\alpha_{1}(G)=|E(G)|$. Let $c(G, x)$ be the generating function of the sequence $\left(\alpha_{i}(G)\right)_{i=0}^{\infty}$, that is,

$$
c(G, x)=\sum_{i \geq 0} \alpha_{i}(G) x^{i} .
$$

For finite graphs $G, c(G, x)$ is a polynomial and we call it the cube polynomial of $G$. For instance, let $T$ be a tree on $n$ vertices, then $c(T, x)=(n-1) x+n$. Note also that $c\left(Q_{n}, x\right)=(x+2)^{n}$ and that

$$
\alpha_{k}(G)=\frac{c^{(k)}(G, 0)}{k !} .
$$

A cover $\mathcal{C}$ of a graph $G$ is a set of induced subgraphs $\mathcal{C}=\left\{G_{1}, \ldots, G_{n}\right\}$ of $G$ such that $G=G_{1} \cup G_{2} \cup \cdots \cup G_{n}$. We say that a cover $\mathcal{C}$ is cubical, if every induced hypercube of $G$ is contained in at least one of the graphs of $\mathcal{C}$. For any subset $\mathcal{A} \subseteq \mathcal{I}_{n}$, let $G_{\mathcal{A}}$ be the intersection (possibly empty and possibly disconnected) of the graphs $G_{i}(i \in \mathcal{A})$.

Proposition 1 Let $\mathcal{C}=\left\{G_{1}, \ldots, G_{n}\right\}$ be a cubical cover of a graph $G$. Then,

$$
c(G, x)=\sum_{\mathcal{A} \subseteq \mathcal{I}_{n}}(-1)^{|\mathcal{A}|-1} c\left(G_{\mathcal{A}}, x\right) .
$$

Proof. For $i \geq 0$ and $j \in\{1,2, \ldots, n\}$, let $A_{i}^{j}$ be the set of induced $i$-cubes of the graph $G_{j}$. Then $\alpha_{i}(G)=\left|A_{i}^{1} \cup A_{i}^{2} \cup \cdots \cup A_{i}^{n}\right|$. Hence, by (4), we infer

$$
\alpha_{i}(G)=\sum_{\mathcal{A} \subseteq \mathcal{I}_{n}}(-1)^{|\mathcal{A}|-1}\left|\bigcap_{j \in \mathcal{A}} A_{i}^{j}\right| .
$$

In addition, $c\left(G_{\mathcal{A}}, x\right)=\sum_{i \geq 0}\left|\cap_{j \in \mathcal{A}} A_{i}^{j}\right| x^{i}$. Therefore,

$$
\begin{aligned}
c(G, x) & =\sum_{i \geq 0} \alpha_{i}(G) x^{i} \\
& =\sum_{i \geq 0}\left(\sum_{\mathcal{A} \subseteq \mathcal{I}_{n}}(-1)^{|\mathcal{A}|-1}\left|\bigcap_{j \in \mathcal{A}} A_{i}^{j}\right|\right) x^{i} \\
& =\sum_{\mathcal{A} \subseteq \mathcal{I}_{n}} \sum_{i \geq 0}\left((-1)^{|\mathcal{A}|-1}\left|\bigcap_{j \in \mathcal{A}} A_{i}^{j}\right|\right) x^{i} \\
& =\sum_{\mathcal{A} \subseteq \mathcal{I}_{n}}(-1)^{|\mathcal{A}|-1} c\left(G_{\mathcal{A}}, x\right) .
\end{aligned}
$$

Because of Proposition 1 we say that a function $f: \mathcal{G} \times \mathbb{R} \rightarrow \mathbb{R}$ has the amalgamation property if

$$
f(G, x)=\sum_{\mathcal{A} \subseteq \mathcal{I}_{n}}(-1)^{|\mathcal{A}|-1} f\left(G_{\mathcal{A}}, x\right) .
$$


whenever $\left\{G_{1}, \ldots, G_{n}\right\}$ is a cubical cover of $G$.

As hypercubes are the simplest Cartesian product graphs, the cube polynomial should behave nicely with respect to the Cartesian product. Indeed, observe that an induced $r$-cube of $G \square H$ is uniquely representable as $Q_{s} \square Q_{r-s}$, where $Q_{s}$ is an induced $s$-cube of $G$ and $Q_{r-s}$ an induced $(r-s)$-cube of $H$. Hence, for every $k \geq 0$,

$$
\alpha_{k}(G \square H)=\sum_{i=0}^{k} \alpha_{i}(G) \alpha_{k-i}(H) .
$$

From here we easily conclude:

Proposition 2 For any graphs $G$ and $H$,

$$
c(G \square H, x)=c(G, x) c(H, x) .
$$

Observe that the identity $|E(G \square H)|=|E(G)||V(H)|+|E(H)||V(G)|$ immediately follows from Proposition 2. We say that a function $f: \mathcal{G} \times \mathbb{R} \rightarrow \mathbb{R}$ has the product property if for any graphs $G$ and $H$,

$$
f(G \square H, x)=f(G, x) f(H, x) .
$$

Let $G$ be a connected graph. The expansion $G^{*}$ of $G$ with respect to the cubical cover $\left\{G_{1}, G_{2}\right\}$ of $G$ is the graph constructed as follows. Let $G_{i}^{*}$ be an isomorphic copy of $G_{i}$, for $i=1,2$, and, for any vertex $u$ of $G_{0}=G_{1} \cap G_{2}$, let $u_{i}$ be the corresponding vertex in $G_{i}^{*}$. Then $G^{*}$ is obtained from the disjoint union of $G_{1}^{*}$ and $G_{2}^{*}$, where for each $u$ of $G_{0}$ the vertices $u_{1}$ and $u_{2}$ are joined by an edge. (Note that, since the cover is cubical, a vertex of $G_{1} \backslash G_{2}$ cannot be adjacent to a vertex of $G_{2} \backslash G_{1}$. Note also that the intersection $G_{1} \cap G_{2}$ is non-empty.)

It is easy to prove the following claim. Just observe that via the expansion the subgraph isomorphic to $G_{0} \square K_{2}$ gives rise to new/larger hypercubes.

Proposition 3 Let $G^{*}$ be a graph constructed by the expansion with respect to the cubical cover $\left\{G_{1}, G_{2}\right\}$ (over $\left.G_{0}\right)$. Then $c\left(G^{*}, x\right)=c\left(G_{1}, x\right)+c\left(G_{2}, x\right)+x c\left(G_{0}, x\right)$.

Because of this result, the following definition seems reasonable. A function $f: \mathcal{G} \times$ $\mathbb{R} \rightarrow \mathbb{R}$ has the expansion property if

$$
f\left(G^{*}, x\right)=f\left(G_{1}, x\right)+f\left(G_{2}, x\right)+x f\left(G_{0}, x\right),
$$

whenever $G^{*}$ is the expansion with respect to the cubical cover $\left\{G_{1}, G_{2}\right\}$ (over $G_{0}$ ).

Theorem 4 Let $f: \mathcal{G} \times \mathbb{R} \rightarrow \mathbb{R}$ be a function with the amalgamation and the expansion property. Then for any graph $G$,

$$
f(G, x)=f\left(Q_{0}, x\right) c(G, x) .
$$


Proof. The proof is by induction on the number of vertices of a graph. The $n$-cube $Q_{n}$, $n \geq 1$, can be obtained as the expansion of the cubical cover $\left\{Q_{n-1}, Q_{n-1}\right\}$ (over $Q_{n-1}$ ). Now, by the expansion property we have $f\left(Q_{n}, x\right)=(2+x) f\left(Q_{n-1}, x\right)$. Hence,

$$
f\left(Q_{n}, x\right)=(2+x)^{n} f\left(Q_{0}, x\right)=c\left(Q_{n}, x\right) f\left(Q_{0}, x\right) .
$$

Suppose now that $G$ is not a hypercube. Let $\mathcal{C}$ be the set comprising of the graphs $G-v$ for every $v \in V(G)$. Since $G$ is not a hypercube, $\mathcal{C}$ is a cubical cover and every graph of $\mathcal{C}$ is smaller than $G$. Using amalgamation property for $c$, and the induction hypothesis for graphs $G_{i}$, we conclude that

$$
\begin{aligned}
f(G, x) & =\sum_{\mathcal{A} \subseteq \mathcal{I}_{n}}(-1)^{|\mathcal{A}|-1} f\left(G_{\mathcal{A}}, x\right) \\
& =\sum_{\mathcal{A} \subseteq \mathcal{I}_{n}}(-1)^{|\mathcal{A}|-1} f\left(Q_{0}, x\right) c\left(G_{\mathcal{A}}, x\right) \\
& =f\left(Q_{0}, x\right) c(G, x) .
\end{aligned}
$$

From the above proof we can also deduce that if $f$ has the amalgamation property and $f\left(Q_{n}, x\right)=(x+2)^{n}$ holds for every $n \in \mathbb{N}_{0}$, then $f \equiv c$.

In the case of median graphs we can strengthen the result of Theorem 4. Recall that Mulder $[12,13]$ proved that a graph is a median graph if and only if it can be obtained from $K_{1}$ by a sequence of expansions in which $G_{0}=G_{1} \cap G_{2}$ is always convex, cf. also [11, 14].

Corollary $\mathbf{5}$ Let $f: \mathcal{M} \times \mathbb{R} \rightarrow \mathbb{R}$ be a function with the expansion property. Then,

$$
f(G, x)=f\left(Q_{0}, x\right) c(G, x) .
$$

If, in addition, $f$ has the product property, then either $f \equiv 0$ or $f \equiv c$.

Proof. Let $f$ has the expansion property. The proof of the first assertion is by induction on the number of expansion steps needed to obtain a median graph. The conclusion is clear for $G=K_{1}$. Let now $G^{*}$ be a median graph obtained by a (convex) expansion with respect to the cubical cover $\left\{G_{1}, G_{2}\right\}$ (over $G_{0}$ ). Then,

$$
f\left(G^{*}, x\right)=f\left(G_{1}, x\right)+f\left(G_{2}, x\right)+x f\left(G_{0}, x\right) .
$$

From the induction hypothesis we obtain

$$
\begin{aligned}
f\left(G^{*}, x\right) & =f\left(Q_{0}, x\right) c\left(G_{1}, x\right)+f\left(Q_{0}, x\right) c\left(G_{2}, x\right)+x f\left(Q_{0}, x\right) c\left(G_{0}, x\right) \\
& =f\left(Q_{0}, x\right)\left(c\left(G_{1}, x\right)+c\left(G_{2}, x\right)+x c\left(G_{0}, x\right)\right) \\
& =f\left(Q_{0}, x\right) c\left(G^{*}, x\right) .
\end{aligned}
$$

If $f$ also has the product property, then, since $Q_{0} \square Q_{0}=Q_{0}$, we have $f\left(Q_{0}, x\right)=$ $\left[f\left(Q_{0}, x\right)\right]^{2}$. Thus either $f\left(Q_{0}, x\right)=0$ or $f\left(Q_{0}, x\right)=1$. Combining this with the fact that $f(G, x)=f\left(Q_{0}, x\right) c(G, x)$ completes the proof. 


\section{Derivation graphs of median graphs}

Let $F$ be a $\Theta$-class of a median graph and let $e=u v \in F$. Then it is well-known that $F$ forms a matching. Moreover, the ends of edges of $F$ that are closer to $u$ than to $v$ induce a median graph isomorphic to the subgraph induced by the ends of edges of $F$ that are closer to $v$ than to $u$. We denote this median subgraph by $U_{e}$. Let $\mathcal{F}(G)$ be the set of edges consisting of representatives of the $\Theta$-classes of $G$. Then we define the derivation of a median graph $G$ as the graph

$$
\partial G=\bigcup_{e \in \mathcal{F}(G)} U_{e},
$$

that is, as the disjoint union of the graphs $U_{e}, e \in \mathcal{F}(G)$. (Note that transitivity of $\Theta$ implies that the graph $\partial G$ is well-defined.) For instance, $\partial\left(P_{n} \square P_{m}\right)$ is the disjoint union of $n-1$ copies of the path $P_{m}$ and $m-1$ copies of the path $P_{n}$.

The reason for calling the graph $\partial G$ "the derivation" of $G$ is the following property.

Proposition 6 (Derivation property) Let $G$ be a median graph. Then,

$$
c^{\prime}(G, x)=c(\partial G, x) .
$$

Proof. Note that for any $Q_{n}$ in $G$ its edges lie in $n \Theta$-classes $(n \geq 1)$, and the corresponding graphs $U_{e}$ induce $(n-1)$-cubes - altogether there are $n Q_{n-1}$ 's for each $n$-cube of $G$. Hence, $n \alpha_{n-1}(G)$ equals the number of $(n-1)$-cubes of $\partial G$. In other words:

$$
c^{\prime}(G, x)=\sum_{e \in \mathcal{F}} c\left(U_{e}, x\right)=c(\partial G, x)
$$

We say that a function $f: \mathcal{M} \times \mathbb{R} \rightarrow \mathbb{R}$ has the derivation property if

$$
f^{\prime}(G, x)=f(\partial G, x)
$$

We next prove that the cube polynomial is the only function on median graphs with the derivation property such that its value in $x=0$ equals the number of vertices.

Theorem 7 Let $f: \mathcal{M} \times \mathbb{R} \rightarrow \mathbb{R}$ be a function with the derivation property, such that $f(G, 0)=|V(G)|$. Then $f \equiv c$.

Proof. First, since $Q_{0}$ has no $\Theta$-classes, $\partial Q_{0}$ is empty. Thus $f\left(Q_{0}, x\right)$ is a constant, and $f\left(Q_{0}, 0\right)=1$ implies $f\left(Q_{0}, x\right)=1$. Hence $f\left(Q_{0}, x\right)=c\left(Q_{0}, x\right)$.

The proof proceeds by induction on the number of vertices of a median graph. Suppose that for any median graph $H$ with less than $k$ vertices we have $f(H, x)=c(H, x)$, and let $G$ have $k$ vertices. Recall that for every $e \in E(G)$, the graph $U_{e}$ is a median graph. Since $U_{e}$ has less than $k$ vertices, $f\left(U_{e}, x\right)=c\left(U_{e}, x\right)$ holds by the induction assumption. 
By the derivation properties of $c$ and $f$ we infer that $f^{\prime}(G, x)=c^{\prime}(G, x)$. Therefore $f(G, x)=c(G, x)+C$, where $C$ is a constant. Since $f(G, 0)=c(G, 0)$, we obtain that $C=0$, and therefore $f \equiv c$.

Let us denote by $\mathcal{M}^{*}$ the class of all graphs whose connected components are median graphs. Thus, $G \in \mathcal{M}^{*}$ can be written as $G=G_{1} \cup G_{2} \cup \cdots \cup G_{s}$, where each $G_{i}$ is a median graph. Then we can extend the concept of the derivation graph to the graphs from $\mathcal{M}^{*}$ by setting $\partial G=\partial G_{1} \cup \partial G_{2} \cup \cdots \cup \partial G_{s}$ and also define the higher derivations in the following way. For $k \geq 0$, set

$$
\partial^{k} G= \begin{cases}\partial\left(\partial^{k-1} G\right) & k \geq 1 \\ G & k=0 .\end{cases}
$$

Then one can easily extend Proposition 6 to the graphs from $\mathcal{M}^{*}$ as well as generalize it to the higher derivatives in the following way:

$$
c^{(k)}(G, x)=c\left(\partial^{k} G, x\right) .
$$

\section{Relations for median graphs}

Throughout this section let $G$ be a median graph with $k \Theta$-classes. Denote by the $\theta_{s}(G)$ $(s \geq 0)$ the number of components in the graph $\partial^{s} G$. Thus, $\theta_{0}(G)=1$ and $\theta_{1}(G)=k$. Recall that a graph is a median graph if and only if it can be obtained from hypercubes by a sequence of convex amalgamations, a result due to Bandelt and van de Vel [3], cf. also $[11,19]$.

Proposition 8 Let $G$ be a median graph and $s \geq 0$. Then,

$$
\theta_{s}(G)=c^{(s)}(G,-1) .
$$

Proof. The proof is by induction on $s$, and afterwards by induction on the number of vertices of a median graph. Suppose first that $s=0$. If $G \cong Q_{n}(n \geq 0)$ then $c\left(Q_{n},-1\right)=1=\theta_{0}\left(Q_{n}\right)$. Now assume that $G$ is the amalgam of $G_{1}$ and $G_{2}$ over $G_{0}$. Then, by the induction assumption,

$$
c(G,-1)=c\left(G_{1},-1\right)+c\left(G_{2},-1\right)-c\left(G_{0},-1\right)=1+1-1=1=\theta_{0}(G) .
$$

Suppose now that the claim holds for all integers smaller than $s(s \geq 1)$ and for all median graphs with less vertices than $G$. Since $c^{(s+1)}(G, x)=\sum_{e \in \mathcal{F}(G)} c^{(s)}\left(U_{e}, x\right)$ we can use the induction hypothesis for graphs $U_{e}$ and derive

$$
c^{(s+1)}(G,-1)=\sum_{e \in \mathcal{F}(G)} \theta_{s}\left(U_{e}\right)=\theta_{s+1}(G) .
$$


Theorem 9 Let $G$ be a median graph and $s \geq 0$. Then,

$$
c^{(s)}(G, x+1)=\sum_{i \geq s} \frac{c^{(i)}(G, x)}{(i-s) !}
$$

and

$$
c^{(s)}(G, x)=\sum_{i \geq s} \frac{(-1)^{i-s}}{(i-s) !} c^{(i)}(G, x+1) .
$$

Proof. The proof of the first equality is by induction on the number of amalgamation steps. Suppose first that $G \cong Q_{n}$. Since $c\left(Q_{n}, x\right)=(x+2)^{n}$ it follows that $c^{(s)}\left(Q_{n}, x+1\right)=$ $\frac{n !}{(n-s) !}(x+3)^{n-s}$. Using binomial formula we obtain:

$$
\begin{aligned}
c^{(s)}\left(Q_{n}, x+1\right) & =\frac{n !}{(n-s) !} \sum_{j=0}^{n-s}\left(\begin{array}{c}
n-s \\
j
\end{array}\right)(x+2)^{j} \\
& =\sum_{j=0}^{n-s} \frac{n !}{(n-s) !} \cdot \frac{(n-s) !}{j !(n-s-j) !}(x+2)^{j} \\
& =\sum_{j \geq 0} \frac{c^{(s+j)}\left(Q_{n}, x\right)}{j !} \\
& =\sum_{i \geq s} \frac{c^{(i)}\left(Q_{n}, x\right)}{(i-s) !}
\end{aligned}
$$

and so the desired formula follows. If $G$ is not a hypercube then it can be obtained by an amalgamation of $G_{1}$ and $G_{2}$ over $G_{0}$. By the induction hypothesis we deduce:

$$
\begin{aligned}
c^{(s)}(G, x+1) & =c^{(s)}\left(G_{1}, x+1\right)+c^{(s)}\left(G_{2}, x+1\right)-c^{(s)}\left(G_{0}, x+1\right) \\
& =\sum_{i \geq s} \frac{1}{(i-s) !}\left(c^{(i)}\left(G_{1}, x\right)+c^{(i)}\left(G_{2}, x\right)-c^{(i)}\left(G_{0}, x\right)\right) \\
& =\sum_{i \geq s} \frac{c^{(i)}(G, x)}{(i-s) !}
\end{aligned}
$$

This proves the first relation.

The second equality can be proved in a similar way. Alternatively, one can write down the first equality for every $0 \leq s \leq r$, where $r$ is the dimension of a largest hypercube of $G$, and invert the obtained system of equations.

Corollary 10 Let $G$ be a median graph, $\alpha_{i}=\alpha_{i}(G)$ the number of induced $i$-cubes in $G$, and $\theta_{i}=\theta_{i}(G)$ the number of components in $\partial^{i} G$. Then for every $s \in \mathbb{N}_{0}$,

$$
\text { (a) } \alpha_{s}=\frac{1}{s !} \sum_{i \geq s} \frac{\theta_{i}}{(i-s) !} \quad \text { and } \quad \theta_{s}=s ! \sum_{i \geq 0}(-1)^{i-s}\left(\begin{array}{l}
i \\
s
\end{array}\right) \alpha_{i}
$$


(b) $\sum_{i \geq 0}(-1)^{i} 2^{i} \alpha_{i}=\sum_{i \geq 0}(-1)^{i} \frac{\theta_{i}}{i !}$.

Proof. The first formula of $(a)$ is obtained by setting $x=-1$ in relation (11).

In order to prove the second relation of $(a)$, we set $x=-1$ in relation (12) to get

$$
(-1)^{s} c^{(s)}(G,-1)=\sum_{i \geq s}(-1)^{i} \frac{c^{(i)}(G, 0)}{(i-s)} .
$$

Therefore

$$
(-1)^{s} \frac{c^{(s)}(G,-1)}{s !}=\sum_{i \geq s}(-1)^{i} \frac{c^{(i)}(G, 0)}{s !(i-s) !} .
$$

Now, using Proposition 8 and (5), we obtain

$$
(-1)^{s} \frac{\theta_{s}}{s !}=\sum_{i \geq s}(-1)^{i}\left(\begin{array}{l}
i \\
s
\end{array}\right) \alpha_{i} .
$$

Finally note that $\left(\begin{array}{l}i \\ s\end{array}\right)=0$ if $i<s$.

In order to obtain the relation (b) just sum up equalities from the first formula of $(a)$ and use basic properties of binomial coefficients.

\section{References}

[1] S. P. Avann, Metric ternary distributive semi-lattices, Proc. Amer. Math. Soc. 12 (1961) 407-414.

[2] H.J. Bandelt, Retracts of hypercubes, J. Graph Theory 8 (1984) 501-510.

[3] H.-J. Bandelt and M. van de Vel, Superextensions and the depth of median graphs, J. Combin. Theory Ser. A 57 (1991) 187-202.

[4] D. Djoković, Distance preserving subgraphs of hypercubes, J. Combin. Theory Ser. B 14 (1973) 263-267.

[5] D. Duffus and I. Rival, Graphs orientable as distributive lattices, Proc. Amer. Math. Soc. 88 (1983) 197-200.

[6] C. D. Godsil, Algebraic Combinatorics (Chapman and Hall, New York, 1993).

[7] W. Imrich and S. Klavžar, Product Graphs: Structure and Recognition (John Wiley \& Sons, New York, 2000).

[8] W. Imrich, S. Klavžar, and H. M. Mulder, Median graphs and triangle-free graphs, SIAM J. Discrete Math. 12 (1999) 111-118. 
[9] S. Klavžar and R. Škrekovski, On median graphs and median grid graphs, Discrete Math. 219 (2000) 287-293.

[10] S. Klavžar, H. M. Mulder, and R. Škrekovski, An Euler-type formula for median graphs, Discrete Math. 187 (1998) 255-258.

[11] S. Klavžar and H. M. Mulder, Median graphs: characterizations, location theory and related structures, J. Combin. Math. Combin. Comp. 30 (1999) 103-127.

[12] H. M. Mulder, The structure of median graphs, Discrete Math. 24 (1978) 197-204.

[13] H. M. Mulder, The Interval Function of a Graph (Mathematical Centre Tracts 132, Mathematisch Centrum, Amsterdam, 1980).

[14] H.M. Mulder, The expansion procedure for graphs, in: R. Bodendiek ed., Contemporary Methods in Graph Theory, B.I.-Wissenschaftsverlag, Mannheim/Wien/Zürich, 1990, 459-477.

[15] L. Nebeský, Median graphs, Comment. Math. Univ. Carolinae 12 (1971) 317-325.

[16] R. Škrekovski, Two relations for median graphs, Discrete Math. 226 (2001) 351-353.

[17] P. S. Soltan and V. D. Chepoi, Solution of the Weber problem for discrete median metric spaces (Russian), Trudy Tbiliss. Mat. Inst. Razmadze Akad. Nauk Gruzin. SSR 85 (1987) 52-76.

[18] C. Tardif, On compact median graphs, J. Graph Theory 23 (1996) 325-336.

[19] M. van de Vel, Theory of Convex Structures (North Holland, Amsterdam, 1993).

[20] P. Winkler, Isometric embeddings in products of complete graphs, Discrete Appl. Math. 7 (1984) 221-225. 